\title{
Anterior Myocardial Infarction Associated with Myocardial Bridging in a Young Man
}

\author{
Ngow $\mathrm{HA}^{\mathrm{a}}$ and Wan Khairina WMN \\ aDepartment of Internal Medicine, 'bepartment of Paediatrics, \\ Kulliyyah of Medicine, International Islamic University Malaysia
}

\begin{abstract}
:
A 43-year-old man presented with acute extensive anterior ST-segment elevation myocardial infarction. During coronary angiogram, a segment of myocardial bridging was noted in the mid-segment of left anterior descending artery. The association of myocardial bridging and an anterior ST segment elevation is rarely reported in the medical literature. Myocardial bridging is caused by systolic compression of a coronary artery by overlying myocardium tissue. It is a rare coronary artery anomaly, which usually has a benign prognosis despite some case reports of myocardial ischemia leading to myocardial infarction, lethal arrhythmias and sudden cardiac death. We report one such case of myocardial bridging that was complicated with acute extensive anterior myocardial infarction.
\end{abstract}

KEYWORDS: Myocardial infarction, myocardial bridging, sudden cardiac death, coronary artery, anomaly

\section{INTRODUCTION}

The major coronary arteries are located on the subepicardial region. When a segment of the coronary artery is embedded in the myocardium, it is termed myocardial bridging. In these patients, this anomaly resulted in temporary systolic coronary artery narrowing. A recent report had found that myocardial bridging affects blood flow in both systole and diastole with significant hemodynamic changes during diastole. ${ }^{1}$ Myocardial bridging has generally been considered as a benign congenital anomaly of the coronary arteries with the attending hazard of myocardial infarction. Reyman first reported it in 1737 and thereafter by Black in $1805 .^{2}$ With the recent advancement of interventional cardiology, intravascular ultrasound and Doppler flow wire have shown that this non-atherosclerotic bridging vessel can produce a marked reduction in the luminal cross sectional area throughout ventricular systole as well as the diastole phase resulting in severe coronary flow limitation. ${ }^{3}$ This discovery help to postulate that myocardial bridging can be a substrate for myocardial ischemia and its related event, myocardial infarction. We report an interesting case which presented acutely with anterior STsegment elevation myocardial infarction associated with this rare anomaly.

\section{CASE REPORT}

A 43-year-old Indian man presented to our casualty with angina pectoris for 2 hours duration. He was a nondiabetic, normotensive and was not known to have atherosclerotic coronary artery disease. He did not take any form of medications or recreational drugs. He was a regular chronic smoker (30 packs/year) and had history of casual alcohol intake. His father had coronary artery disease and died at the age of 60 . The patient was normo-tensive with blood pressure of $108 / 64 \mathrm{mmHg}$. His fasting blood sugar was $5.3 \mathrm{mmol} / \mathrm{L}$ and the LDL cholesterol was $2.84 \mathrm{mmol} / \mathrm{L}$. The electrocardiogram revealed a 7-mm ST-segment elevation in the precordial leads associated with an elevated myocardial marker (Figure 1). The Creatinine Kinase level was $708 \mathrm{u} / \mathrm{L}(\mathrm{N}: 24-204 \mathrm{u} / \mathrm{L})$ and Cardiac Troponin $\mathrm{T}$ was $2.08 \mathrm{ug} / \mathrm{L}(\mathrm{N}<0.1 \mathrm{ug} / \mathrm{L})$. The echocardiogram showed good left ventricular function (Ejection Fraction, $\mathrm{EF}=61 \%$ ) without any regional wall motion abnormality. The patient was given intravenous rtPA, antiplatelet and antianginal. The fibrinolytic agent was given 20 minutes after admission. A repeat electrocardiogram showed complete resolution of the ST segment 1 hour post- thrombolysis (Figure 2).
Corresponding author

Harris Ngow Abdullah

Internal Medicine Department,

International Islamic University Malaysia,

e-mail: harrisngow@gmail.com 

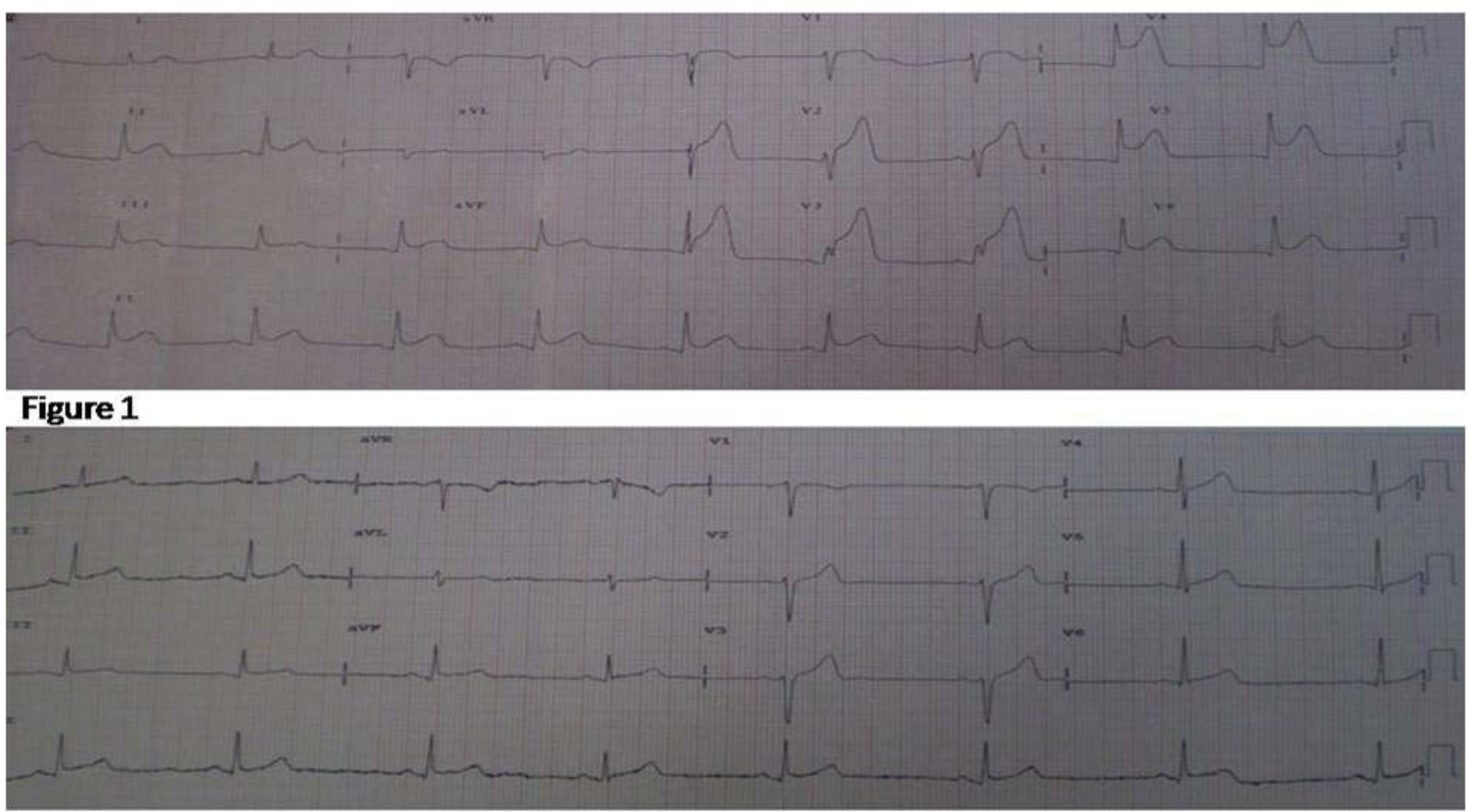

Figure 2

The electrocardiogram of this patient shows extensive anterior ST- elevation myocardial infarction(Figure 1) followed by ST segment resolution post fibrinolysis. (Figure 2)

Coronary angiogram revealed a long segment (approximately $30 \mathrm{~mm}$ ) myocardial bridging at the mid portion of the left anterior descending artery without stenosis or thrombus. However, a $30 \%-40 \%$ atherosclerotic plaque was seen proximal to the myocardial bridge. (Figure 3 and Figure 4) We postulated that his myocardial infarction was due to the adverse interplay of his smoking habits, mild atherosclerotic plaque and the pre-existing myocardial bridging. He was managed with optimal medical therapy with beta blocker and calcium channel blocker. Two years later, the patient remained well and asymptomatic. His latest dobutamine stress echocardiography was normal.

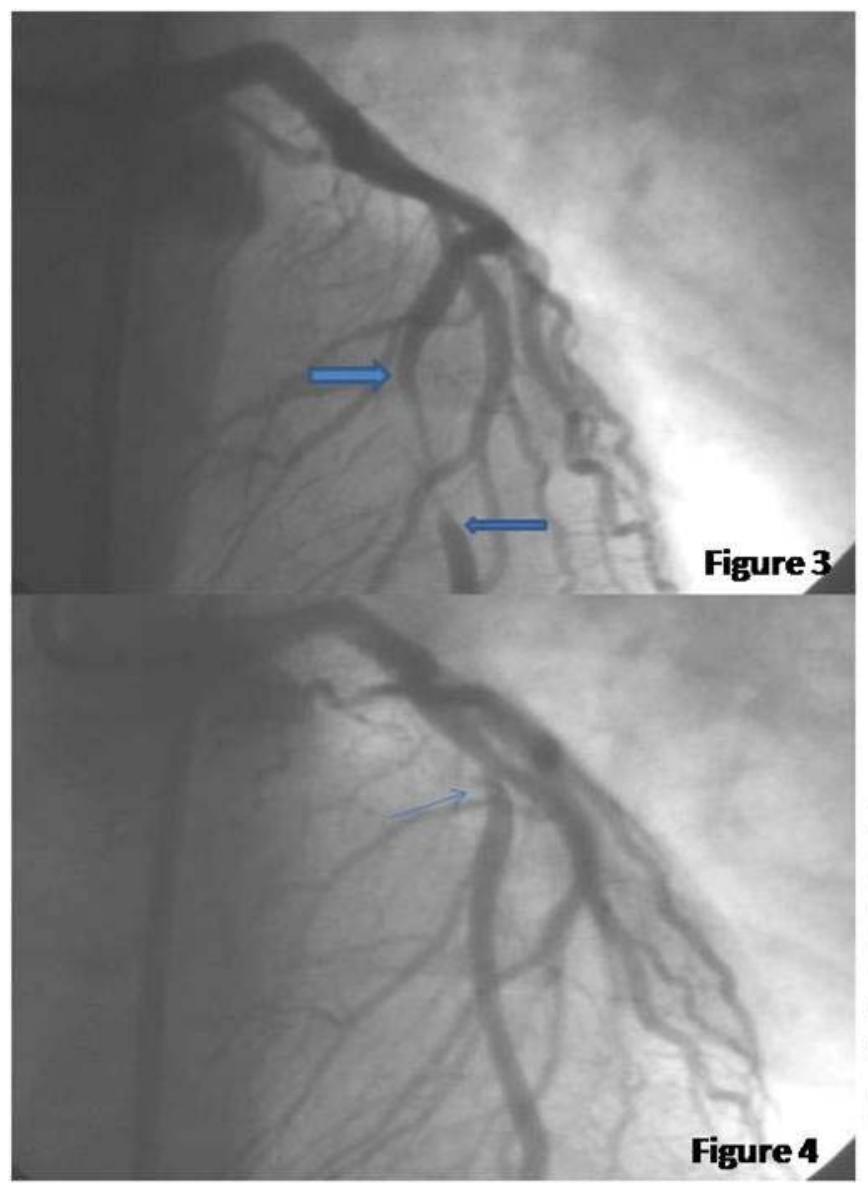

\section{Figure 3}

Left coronary injection in the anteroposterior (AP) cranial view shows the bridge segment in the left anterior descending artery during systole. (Length indicated by arrow)

\section{Figure 4}

Left coronary injection in the anteroposterior (AP) cranial view shows the bridge segment in the left anterior descending artery during diastole. The thin arrow shows the atherosclerotic plaque proximal to themyocardial bridge 


\section{DISCUSSION}

The reported incidence of myocardial bridging is $0.5 \%$ to $16 \%$ for patients undergoing coronary angiogram and the prevalence from autopsy series can range from $15 \%$ to $85 \%$ depend on the study performed. ${ }^{1,4-5}$ Evidence derived from intravascular ultrasound and Doppler studies had shown that myocardial bridges especially the deep and long segment, could contribute to haemodynamic burden on the coronaries. Symptomatic angina has been reported in 50\% to $70 \%$ of patients confirmed with isolated myocardial bridges. ${ }^{6}$ Autopsy study by Ramos et al had observed that the severity of atherosclerosis in the proximal portion segment of the myocardial bridging was greater compared to the distal segment of the vessel. ${ }^{7}$ This result was also similarly found in $\mathrm{Ge}$ et al series. They performed intravascular ultrasound in 14 patients with angiographic evidence of myocardial bridging in the left anterior descending artery. Atherosclerotic plaques were found in the proximal segment in 12 out of the 14 patients. There was no atherosclerotic lesion detected in the myocardial bridging itself or the distal segment in eight patients, in whom the intravascular ultrasound catheter was successfully advanced across the entire myocardial bridging. ${ }^{3}$ In our patient, the angiographic findings were consistent with their result. There was a $30 \%$ to $40 \%$ atherosclerotic plague proximal to the myocardial bridging. The proximal segment frequently shows atherosclerotic plaque formation compared to the tunnel vessel. Cellular and ultrastructural studies showed that in contrast to the proximal and distal segment, the tunnelled segment was lacking of foam cells and modified contractile smooth muscle cells thus protecting it from atherosclerosis. In addition, alteration to shear stress and local chemokines production resulted in atheroprotective effect of the tunnelled vessel. ${ }^{1}$ The possibility of thrombus formation as a result of proximal plaque rupture could not be ruled out in our patient. Intravascular ultrasound or optical coherence tomography might be useful to visualise the ruptured plaque, however, these investigations were not performed.

Although it remains elusive why atherosclerosis is confined mainly in the proximal segment, Ge et al had suggested that local wall stress and subsequent endothelial injury may result in local atherosclerosis or thrombus formation proximal to the myocardial bridging. ${ }^{3}$

The mild proximal atherosclerotic plaque however does not by itself lead to ischemia. The induction of ischemia by a myocardial bridge has been demonstrated through various mechanisms which include thrombus formation, vasospasm, endothelial dysfunction or impaired coronary flow reserve. ${ }^{1}$ It is hypothesized that the endothelial cell damage with continuous mechanical stress predisposes the tunnelled vessel to vasospasm and premature atherosclerosis on the segment proximal to the myocardial bridge leading to ischemia and angina. Moreover, the diastolic "Spike and Dome" phenomenon of the coronary blood flow velocity that is typically seen on Colour Doppler interrogation is indicative of the maximum perfusion pressure coincides with a persistently contracted vessel surface area. ${ }^{8}$ This gives rise to a steep pressure gradient across the myocardial bridging and subsequently results in a sudden reduce coronary reserve flow and ischemia.

In tachycardic state where diastole phase is shortened, the delay in the diastolic lumen gain is more pronounced. This is also true in condition where myocardial contractility is increased, such as in strenuous exercise or extreme exertion. The illustrated case highlights the fact that myocardial bridging can lead to major ischemic events associated with smoking though an element of associated vasospasm cannot be convincingly ruled out. In this patient no other identifiable risks could explain the myocardial infarction, besides family history, smoking and myocardial bridging as the patient is normo-tensive and normoglycaemic. His total cholesterol was $4.49 \mathrm{mmol} / \mathrm{L}$ and LDL cholesterol was $2.84 \mathrm{mmol} / \mathrm{L}$. Smoking might lead to thrombus formation, associated with coronary spasm, increase platelet aggregation and blood coagulability. The effect of smoking compounded by the existence of myocardial bridging might explain the myocardial event.

Several intravascular ultrasound studies had shown that vessel compression in myocardial bridge is not limited to systole but also persist into diastole, which is the predominant phase of myocardial perfusion. 1 Therefore, even in the absence of critical coronary artery disease, this mechanism explained the diminished oxygen delivery to the myocardium in systolic as well as diastolic phase. In our patient, this phenomenon was not demonstrated as the angiography showed that the coronary flow was limited only in the systolic phase. However, with the use of intravascular ultrasound, intracoronary Doppler ultrasound and intracoronary pressure devices, morphological and functional features of myocardial bridge would be better described. 3,9

Therapeutic strategies in symptomatic patients are various. Traditionally, either coronary artery bypass surgery, minimally invasive coronary artery bypass surgery or debridging myotomy was recommended. ${ }^{1}$ However, angioplasty with coronary stents to the tunnel vessel had also been reported to be successful in some cases. ${ }^{10}$ There are still issues arising from stenting such as stent compression and metal fatigue resulting in stent fracture especially in the long-term that may preclude stenting as a standard treatment. In addition, long bridging segment $(>25 \mathrm{~mm})$ predisposes to high risk of stent thrombosis and restenosis, therefore angioplasty should not be recommended in such situation. ${ }^{9}$

In our patient, we aimed for optimal medical treatment with beta blocker and calcium channel blocker. Nitrates should be avoided as they have been shown to increase the angiographic degree of systolic nar- 
rowing and can lead to worsening of myocardial ischemia. ${ }^{1}$ In view of uncertainties regarding the long-term outcome of stenting and high in-stent thrombosis and restenosis rate with long segment $(>25 \mathrm{~mm})$, we do not recommend coronary stenting in such patient. If the patient developed refractory or recurrent coronary events, surgical debridging myotomy may be the appropriate therapeutic option for this patient.

In conclusion, there are myriad of strategies in managing symptomatic myocardial bridging. Generally, the long-term prognosis of patients with isolated myocardial bridging is excellent. The decision on treatment selection mainly depends on the patient's symptoms. Although, generally nitrates are the first line treatment in reducing myocardial ischemia, it can cause worsening angina in myocardial bridging. In patient with refractory symptoms, debridging myotomy is the recommended alternative. In our patient, he continues remained well and asymptomatic for the past 2 years.

\section{REFERENCES:}

1. Garris JB, Kangarloo H, Sample WF. Ultrasonic Diagnosis of Infrahepatic Interruption of the Inferior Vena Cava with Azygos (Hemiazygos) Continuation. Radiology 1980; 134:179-83

2. Dean SM, Tytle TL. Acute right lower extremity iliofemoral deep venous thrombosis secondary to an anomalous inferior vena cava: a report of two cases. Vasc Med 2006; 11:165-9

3. van der Horst RL, Hastreiter AR. Congenital Interruption of the Inferior Vena Cava. Chest 1981; 80:638-40

4. Bass JE, Redwine MD, Kramer LA, Huynh PT, Harris JH, Jr. Spectrum of Congenital Anomalies of the Inferior Vena Cava: Cross-sectional Imaging Findings. Radiographics 2000; 20:639-52

5. Palotas A, Paszt A, Szentpali K, Lazar G. Esophageal cancer complicated with azygos continuation of the inferior vena cava. Interact CardioVasc Thorac Surg 2003; 2:361-3

6. Berdon WE, Baker DH. Plain film findings in azygos continuation of the inferior venacava. Am J Roentgenol 1968; 104:452-7 